\title{
Peak Pressure Gradient
}

National Cancer Institute

\section{Source}

National Cancer Institute. Peak Pressure Gradient. NCI Thesaurus. Code C147155.

The quantitative measurement of the largest pressure gradient across a predetermined point. 